\title{
Achados ecocardiográficos na cardiomiopatia hipertrófica
}

\author{
Echocardiographic findings in hypertrophic cardiomyopathy \\ Hallazgos ecocardiográficos en la miocardiopatía hipertrófica
}

José Lopes Cavalcante Júnior ${ }^{1 *}$, Marlucia do Nascimento Nobre ${ }^{1}$, Anne Elizabeth Andrade Sadala Marques $^{1}$, Andrea Machado Salgado ${ }^{1}$, Marcelo Mello Gonçalves ${ }^{1}$, Antônio Augusto de Souza Marinho², Hudson Laert Machado de Miranda'.

\section{RESUMO}

Objetivo: Demonstrar a importância da ecocardiografia transtorácica no diagnóstico e seguimento dos pacientes com cardiomiopatia hipertrófica. Revisão bibliográfica: A cardiomiopatia hipertrófica caracterizase por um espessamento miocárdico anormal na ausência de condições que o justifiquem, como por exemplo, hipertensão arterial sistêmica e estenose aórtica. Possui diferentes formas de apresentação, sendo a septal assimétrica a mais comum. Pode ocorrer em outras localizações menos frequentes como apical, concêntrica e no ventrículo direito. Alguns casos evoluem com obstrução da via de saída do ventrículo esquerdo, quando o gradiente de pico é superior a $30 \mathrm{mmHg}$ no repouso e $50 \mathrm{mmHg}$ no esforço ou após manobra de Valsalva. Insuficiência mitral, arritmias, disfunção diastólica e sistólica, são outras complicações que podem estar presentes. Considerações finais: A ecocardiografia transtorácica é o principal exame no diagnóstico desta patologia. Permite identificar e quantificar a gravidade da hipertrofia, suas complicações, além de fornecer importantes dados prognósticos quanto ao risco de morte súbita cardíaca.

Palavras-chave: Ecocardiograma, Cardiomiopatia hipertrófica, Morte súbita cardíaca.

\begin{abstract}
Objective: To demonstrate the importance of transthoracic echocardiography in the diagnosis and follow-up of patients with hypertrophic cardiomyopathy. Bibliographic review: Hypertrophic cardiomyopathy is characterized by an abnormal myocardial thickening in the absence of conditions that justify it, such as systemic arterial hypertension and aortic stenosis. It has different forms of presentation, with the asymmetric septal being the most common. It can occur in other less frequent locations, such as apical, concentric and right ventricle. Some cases evolve with left ventricular outflow tract obstruction, when the peak gradient is greater than $30 \mathrm{mmHg}$ at rest and $50 \mathrm{mmHg}$ on exertion or after Valsalva maneuver. Mitral regurgitation, arrhythmias, diastolic and systolic dysfunction are other complications that may be present. Final considerations: Transthoracic echocardiography is the main diagnostic test for this pathology. It allows identifying and quantifying the severity of hypertrophy, its complications, in addition to providing important prognostic data regarding the risk of sudden cardiac death.
\end{abstract}

Keywords: Echocardiogram, Hypertrophic cardiomyopathy, Sudden cardiac death.

\section{RESUMEN}

Objetivo: Demostrar la importancia de la ecocardiografía transtorácica en el diagnóstico y seguimiento de pacientes con miocardiopatía hipertrófica. Revisión bibliográfica: La miocardiopatía hipertrófica se caracteriza por un engrosamiento miocárdico anormal en ausencia de condiciones que lo justifiquen, como hipertensión arterial sistémica y estenosis aórtica. Tiene diferentes formas de presentación, siendo el septal asimétrico el más común. Puede ocurrir en otras localizaciones menos frecuentes, como apical, concéntrico y ventrículo derecho. Algunos casos evolucionan con obstrucción del tracto de salida del ventrículo izquierdo, cuando el gradiente máximo es mayor de $30 \mathrm{mmHg}$ en reposo y $50 \mathrm{mmHg}$ en el esfuerzo o después de la maniobra de Valsalva. La regurgitación mitral, arritmias, disfunción diastólica y sistólica son otras

1 Universidade Federal do Amazonas (UFAM), Hospital Universitário Getúlio Vargas (HUGV), Manaus - AM.

*E-mail: jlcavalcantejr@gmail.com

${ }^{2}$ Hospital Adventista de Manaus, Manaus - AM.

SUBMETIDO EM: 10/2021 
complicaciones que pueden estar presentes. Consideraciones finales: La ecocardiografía transtorácica es la principal prueba diagnóstica de esta patología. Permite identificar y cuantificar la gravedad de la hipertrofia, sus complicaciones, además de aportar importantes datos pronósticos sobre el riesgo de muerte súbita cardíaca.

Palabras clave: Ecocardiograma, Miocardiopatía hipertrófica, Muerte cardíaca súbita.

\section{INTRODUÇÃO}

A cardiomiopatia hipertrófica $(\mathrm{CMH})$ caracteriza-se pelo espessamento miocárdico inapropriado, ou seja, na ausência de uma condição clínica que o justifique, por exemplo, hipertensão arterial sistêmica importante e estenose aórtica grave (WILLIAMS LK, et al., 2009). Trata-se de uma doença genética autossômica dominante causada por mutações em genes codificadores de proteínas sarcoméricas (LOSI MA, et al., 2010).

É uma das doenças genéticas cardíacas mais comuns, com uma prevalência estimada de 1 caso a cada 500 pessoas (SEMSARIAN C, et al., 2015). Raramente a hipertrofia se desenvolve durante a infância, sendo mais comum o seu aparecimento em adolescentes e adultos jovens. É incomum surgir após a quinta década de vida (MARIAN AJ e BRAUNWALD E, 2017).

Complicações associadas a $\mathrm{CMH}$ são relativamente comuns. Estudos retrospectivos demonstraram uma taxa de ocorrência de fibrilação atrial em torno de $20 \%$, enquanto a prevalência de morte súbita cardíaca ou parada cardiorrespiratória revertida foi de $4 \%$ ). Tais complicações, quando presentes, são marcadores de pior prognóstico nesses pacientes (TURVEY L, et al., 2020.

O eletrocardiograma encontra-se alterado em mais de $90 \%$ dos casos e não existe um achado específico, embora a presença de sinais eletrocardiográficos de sobrecarga ventricular esquerda seja bastante comum (MARON MS, et al., 2017). As alterações localizadas ou difusas da repolarização ventricular também são frequentes (BAZAN SGZ, et al., 2020).

O estudo ecocardiográfico é uma ferramenta fundamental para o diagnóstico, manejo e prognóstico desses pacientes. Sendo assim, o objetivo dessa revisão foi demonstrar a importância da ecocardiografia transtorácica como principal método complementar na avaliação de pacientes com cardiomiopatia hipertrófica, descrevendo os principais achados dessa patologia que podem ser encontrados durante a realização do exame.

\section{REVISÃO BIBLIOGRÁFICA}

\section{Diagnóstico}

Uma avaliação ecocardiográfica sistemática é indicada para os casos suspeitos de $\mathrm{CMH}$ e também em parentes de pacientes com o diagnóstico. A análise deve envolver todos os segmentos do ventrículo esquerdo (VE), com uma varredura da base até o ápice cardíaco. A medida da espessura do miocárdio deve ser feita ao final da diástole e recomenda-se utilizar a janela em eixo curto do VE ao nível da valva mitral, músculos papilares e ápice, para realizar medições sucessivas e confiáveis (DOMINGUEZ F, et al., 2017).

Devem-se evitar medidas realizadas por meio do modo $\mathrm{M}$ na janela paraesternal em eixo longo, uma vez que cortes mais oblíquos podem levar a superestimativa das espessuras septais (ELLIOTT PM, et al., 2014). Além disso, atenção durante a medição da espessura do septo para não incluir elementos associados a ele, como por exemplo, cordas tendíneas, o que implicaria num falso aumento de sua largura e diagnóstico incorreto de CMH (TURVEY L, et al., 2020).

Os padrões de hipertrofia e os volumes cardíacos, que costumam estar normais ou reduzidos, podem ser bem definidos pela ecocardiografia (NAGUEH SF, et al., 2011). A hipertrofia é geralmente assimétrica, de distribuição variável entre os diferentes seguimentos, podendo inclusive atingir o ventrículo direito (VD), mas também pode ser concêntrica. O principal local de acometimento é a parede septal (> $75 \%$ dos casos), sobretudo no seu seguimento basal, mas frequentemente estende-se para a parede lateral, apical ou concêntrico (ELLIOTT PM, et al., 2014). Por vezes pode ser necessário o uso de contraste ecocardiográfico para identificar e definir a extensão de hipertrofia apical (NAGUEH SF, et al., 2011). 
Critérios foram estabelecidos para definir o diagnóstico de $\mathrm{CMH}$ : espessura de qualquer segmento miocárdico > $15 \mathrm{~mm}$; relação entre a espessura do septo interventricular e a parede posterior $>1,3$ em não hipertensos e naqueles considerados hipertensos $>1,5$; espessura miocárdica $>13 \mathrm{~mm}$ em parentes de primeiro grau de pacientes com $\mathrm{CMH}$; em crianças presença de hipertrofia de qualquer segmento miocárdico acima de 2 desvios-padrão que a média prevista para a idade (ELLIOTT PM, et al., 2014; DOMINGUEZ F, et al., 2017).

Algumas literaturas consideram a relação entre o septo interventricular e a parede posterior do ventrículo esquerdo como um critério pouco sensível e específico, visto que várias outras condições podem cursar com esse achado, não sendo possível confirmar o diagnóstico de CMH (ROBINSON A e KRAMER CM, 2018).

A morfologia do septo interventricular pode fornecer informações valiosas quanto à origem da hipertrofia. Um septo reverso correlaciona-se com alta probabilidade de mutações em genes sarcoméricos. Por outro laudo, septo sigmóide tem baixa associação com mutações, podendo estar presente em outras condições, como hipertensão arterial sistêmica e indivíduos idosos (MANDES L, et al., 2020).

Em alguns casos pode haver envolvimento do ventrículo direito. Nessas situações considera-se um VD hipertrofiada quando a espessura de sua parede avaliada na janela subcostal longitudinal for superior a $5 \mathrm{~mm}$ (DOMINGUEZ F, et al., 2017). Outras literaturas consideram como critério diagnóstico para hipertrofia do VD valores maiores que $8 \mathrm{~mm}$. Em virtude das trabeculações presentes em ventrículo direito, o examinador deve ter cuidado para não incluí-las na medição de suas paredes, assim como a gordura epicárdica e pericárdio (MARON MS, et al., 2017).

A forma apical é mais comum na população asiática, muitas vezes com apresentação assintomática. A suspeita é levantada inicialmente após realização de eletrocardiograma, que demonstra ondas T profundas em derivações anteriores. Os seguimentos basais são geralmente poupados, com o espessamento patológico ocorrendo em direção ao ápice (FEIGENBAUM WA e RYAN T, 2012). Foi relatado também que em pacientes com CMH apical, a espessura pode ser inferior a $15 \mathrm{~mm}$. O diagnóstico passa a ser definido quando a relação entre a largura apical e basal for superior a 1,3 (TURVEY L, et al., 2020).

Qualquer exame de imagem cardíaca capaz de definir esses critérios pode ser utilizado para o diagnóstico de $\mathrm{CMH}$, incluindo ecocardiografia, ressonância e tomografia (ELLIOTT PM, et al., 2014). A ressonância magnética do coração pode fornecer informações mais precisas em determinados cenários, como nos casos de acometimento apical, anterolateral e determinadas regiões do septo inferior. Nessas situações, as imagens obtidas por meio da ecocardiografia podem ser limitadas, dificultando o diagnóstico preciso (SEMSARIAN C, et al., 2015).

Em virtude da distribuição geralmente heterogênea da hipertrofia ventricular, as fórmulas habitualmente utilizadas para quantificação da massa miocárdica, podem resultar em erros de medida e não devem ser aplicadas rotineiramente. Vários outros índices foram propostos para minimizar tais erros, mas que ainda precisam ser validados (LOSI MA, et al., 2010).

\section{Avaliação da valva mitral}

Inicialmente acreditava-se que a $\mathrm{CMH}$ era uma doença restrita ao miocárdio ventricular. Atualmente, é de conhecimento que até $59 \%$ dos pacientes com esse diagnóstico apresentam alterações da valva mitral diretamente secundárias as mutações genéticas responsáveis pela etiologia da doença. O movimento anterior sistólico da valva mitral (SAM) está presente em 30-60\% dos pacientes com CMH (MANDES L, et al., 2020).

Essa alteração não é patognomônica, podendo inclusive ser encontrada em pacientes sem cardiopatia. $O$ SAM pode causar obstrução na via de saída do ventrículo esquerdo (VSVE), prolongando o tempo de ejeção e reduzindo o volume sistólico. Forças de arrasto geradas pela contração do VE hipertrofiado deslocam o aparelho valvar mitral em direção a VSVE, resultando em obstrução dinâmica. Além disso, é comum ocorrer falha de coaptação da valva mitral, produzindo regurgitação (NAGUEH SF, et al., 2011).

A insuficiência mitral associada ao SAM tem tipicamente uma orientação inferolateral para dentro do átrio esquerdo. Jatos regurgitantes com direção central ou anterior devem levantar hipótese de doença primária da valva mitral (ELLIOTT PM, et al., 2014). O SAM pode ser facilmente observado também pela técnica unidimensional (modo M) juntamente com a hipertrofia septal (LOSI MA, et al., 2010). 
Várias outras alterações da valva mitral podem ser encontradas em pacientes com $\mathrm{CMH}$. Folhetos valvares mitrais alongados quando comparados a corações normais, são achados comuns em paciente com $\mathrm{CMH}$ obstrutiva. Cordas tendíneas com inserção anômala e músculos papilares deslocados em direção ao septo interventricular, também são importantes fatores presentes e que contribuem para a obstrução da via de saída do ventrículo esquerdo (DOMINGUEZ F, et al., 2017).

\section{Obstrução da via de saída do ventrículo esquerdo}

É definida pela presença de um gradiente de pico na VSVE em repouso, maior ou igual a $30 \mathrm{mmHg}$. O estresse gerado na parede ventricular pela obstrução leva a um remodelamento do ventrículo esquerdo, predispondo à isquemia e arritmias ventriculares graves (STOLL CM, et al., 2021). A existência de obstrução é importante fator prognóstico, pois aumenta o risco de morte súbita cardíaca (MSC) e progressão para insuficiência cardíaca. Os principais mecanismos da obstrução são o estreitamento da VSVE ocasionado pela hipertrofia septal e o SAM (WILLIAMS LK, et al., 2009).

A presença de obstrução deve ser avaliada utilizando-se o mapeamento com doppler colorido, desde a porção média da cavidade ventricular até a VSVE, observando-se a existência de fluxo turbulento. Além disso, o uso do doppler pulsado do ápice cardíaco em direção ao trato de saída, permite localizar o nível anatômico da obstrução, no momento em que ocorrer o fenômeno de aliasing (SUAIDE CES, 2012). Em seguida, por meio do doppler de onda contínua, o gradiente máximo da VSVE é determinado (NAGUEH SF, et al., 2011). Importante levar em conta que a magnitude do gradiente pode ser influenciada por inúmeras variáveis hemodinâmicas, tais como ritmo cardíaco, frequência, pré-carga e pós-carga (ROBINSON A e KRAMER CM, 2018).

Pacientes que não possuem obstrução significativa em repouso devem ser avaliados através de exercício físico ou realização de manobra de Valsalva. Nesses casos, considera-se uma obstrução hemodinamicamente significativa quando maior que $50 \mathrm{mmHg}$. O uso de dobutamina para avaliar obstrução de VSVE não é recomendado, pois pode ser mal tolerada pelo paciente (ELLIOTT PM, et al., 2014).

Cuidados devem ser tomados para não medir erroneamente o fluxo da regurgitação mitral e superestimar o gradiente da VSVE (NAGUEH SF, et al., 2011). A curva de velocidade do fluxo de VSVE nos casos de CMH obstrutiva, possui um pico tardio, assumindo o formato de uma adaga (ROBINSON A e KRAMER CM, 2018). No início da sístole, esse fluxo tem sinal menos intenso, sendo incrementado no final dessa fase do ciclo cardíaco. Por outro lado, o jato da regurgitação mitral já apresenta um pico protossistólico e costuma ter velocidades maiores que a obstrução da VSVE. Frequentemente observamos algum grau de regurgitação aórtica, geralmente leve, decorrente da lesão valvar aórtica provocada pelo fluxo de alta velocidade da VSVE (SUAIDE CES, 2012).

Cerca de $10 \%$ dos pacientes com $\mathrm{CMH}$ apresenta obstrução do segmento médio da cavidade ventricular esquerda. O mecanismo geralmente é a presença de um músculo papilar anômalo ou uma hipertrofia apical acentuada. Esses casos estão muito associados com desenvolvimento de aneurisma apical e costumam ser bastante sintomáticos e refratários ao uso de betabloqueadores e antagonistas dos canais de cálcio (DOMINGUEZ F, et al., 2017).

\section{Avaliação da função sistólica}

Os métodos habituais para avaliação da função sistólica na $\mathrm{CMH}$ (fração de ejeção e fração de encurtamento) geralmente demonstram uma contratilidade normal ou aumentada. Apesar disso, queda na função longitudinal pode ser observada pela redução da velocidade de deslocamento dos anéis mitrais medial e lateral, tanto na sístole quanto na diástole por meio do doppler tecidual (TDI), antes mesmo da presença de hipertrofia evidente (NAGUEH SF, et al., 2011).

O comprometimento na função longitudinal na $\mathrm{CMH}$ coincide com os locais de hipertrofia. A deformidade miocárdica apresenta-se comprovadamente diminuída. Entretanto, mecanismos compensatórios que mantêm a fração de ejeção normal ou aumentada, dão a impressão de boa função sistólica nesses pacientes. Dessa maneira, a fração de ejeção é um parâmetro limitado para a análise da função sistólica, uma vez que se encontra superestimada (SEN-CHOWDHRY S, et al., 2016). 
Em estágios finais, uma minoria dos casos (2-5\%) pode evoluir para disfunção sistólica progressiva, com dilatação e fração de ejeção menor que $50 \%$, conferindo aumento da mortalidade e risco de MSC (NAGUEH SF, et al., 2011). Nesse estágio, o diagnóstico diferencial com cardiomiopatia dilatada pode ser difícil, sendo muitas vezes o conhecimento prévio de hipertrofia no histórico do paciente, a única forma de estabelecer essa diferenciação (SUAIDE CES, 2012).

Dessa maneira a função ventricular hiperdinâmica, característica do início do quadro, pode progredir para uma hipocinesia global. Nessa fase final da doença, o SAM pode não estar mais presente (FEIGENBAUM WA e RYAN T, 2012). Embora o mecanismo para esse tipo de evolução desfavorável ainda não esteja bem elucidado, o gradiente obstrutivo da VSVE provavelmente tem papel importante nesse contexto, levando à dilatação ventricular e elevação das pressões de enchimento (MARON BJ, 2018). Além disso, parece haver uma isquemia microvascular difusa, gerando adelgaçamento das fibras musculares, morte celular e fibrose, contribuindo para a disfunção sistólica estabelecida (DOMINGUEZ F, et al., 2017)

\section{Análise da função diastólica}

Anormalidades no enchimento ventricular estão presentes nos pacientes com $\mathrm{CMH}$, independente da localização e da extensão da hipertrofia (NAGUEH SF, et al., 2011). É um achado decorrente da fibrose intersticial, que faz parte das alterações anatomopatológicas da doença, e da rigidez miocárdica, secundária ao espessamento da parede ventricular (MARIAN AJ e BRAUNWALD E, 2017).

$\mathrm{Na}$ fisiologia normal, o ápice cardíaco se contrai em sentido anti-horário, enquanto a base em sentido horário, semelhante ao que ocorre quando torcemos uma toalha. $\mathrm{Na} \mathrm{CMH}$ a torção do ápice é superior à de um coração normal. No entanto, a distorção na fase diastólica é desorganizada e com atraso, levando à elevação das pressões de enchimento ventricular (SUAIDE CES, 2012).

A disfunção diastólica é uma das principais causas de dispneia nesses pacientes. A velocidade e duração da onda A reversa medida ao nível das veias pulmonares, tem relação significativa com a pressão diastólica final do ventrículo esquerdo, apresentando-se aumentada em amplitude e duração (DOMINGUEZ F, et al., 2017).

O volume do átrio esquerdo (AE) também deve ser considerado para a avaliação da função diastólica, principalmente na ausência de fibrilação atrial e regurgitação mitral significativa, pois são causas de aumento do $A E$ independentemente das pressões de enchimento do VE. É comum pacientes com CMH apresentarem aumento do $A E$, sendo um fator prognóstico de evolução para insuficiência cardíaca e mortalidade cardiovascular, principalmente quando apresenta diâmetros maiores que 48 mm (LOSI MA, et al., 2010).

Outro parâmetro avaliado é a relação E/e' que demonstrou correlação razoável com as pressões de enchimento do VE. Estudos demonstraram redução nas velocidades de deslocamento do anel mitral em ventrículos com relaxamento comprometido (NAGUEH SF, et al., 2011).

Dessa maneira, quatro critérios principais devem avaliados no estudo da função diastólica em pacientes com CMH: E/e'> 14; aumento do volume atrial esquerdo $>34 \mathrm{~mL} / \mathrm{m}^{2}$; onda A reversa com duração $>30$ mseg; velocidade de pico do fluxo da regurgitação tricúspide $>2,8 \mathrm{~m} / \mathrm{s}$. (DOMINGUEZ F, et al., 2017).

Um único critério presente caracteriza a disfunção diastólica grau I. A presença de três ou quatro critérios define a presença de disfunção diastólica grau II. Uma relação $E / A>2$, e' septal e lateral $<7$ e $10 \mathrm{~cm} / \mathrm{s}$ respectivamente, implica em uma disfunção diastólica grau III, ou seja, com um padrão de enchimento restritivo. No caso de dois critérios presentes, a avaliação da função diastólica é inconclusiva (NAGUEH SF, at al., 2016).

\section{Rastreamento ecocardiográfico}

Algumas estratégias de triagem são recomendas para parentes de primeiro grau de pacientes com $\mathrm{CMH}$, de acordo com a idade: < 12 anos: recomendado apenas nos casos de história familiar de MSC por CMH; 12 a 18 - 21 anos: ecocardiografia transtorácica deve ser feita a cada $12-18$ meses; > 18 - 21 anos: realizar triagem a cada 5 anos ou no surgimento de alguma sintomatologia suspeita (QUADER N, et al., 2017). 


\section{Diagnóstico diferencial}

Diversas condições clínicas podem causar hipertrofia ventricular esquerda, tornando-se um desafio para o diagnóstico diferencial com $\mathrm{CMH}$. Situações como hipertensão arterial, estenose aórtica, atletas de alto rendimento, grupos étnicos, raças e gêneros, podem apresentar padrões de hipertrofia semelhantes à $\mathrm{CMH}$. Por exemplo, indivíduos hipertensos africanos podem apresentar espessuras de parede superiores a $15 \mathrm{~mm}$ na ausência de $\mathrm{CMH}$, enquanto esse mesmo padrão se observado em pacientes hipertensos caucasianos, sugere fortemente o diagnóstico da doença. Em geral, nessas condições, o padrão de hipertrofia tende a ser concêntrico, contrastando com o padrão assimétrico da CMH (TURVEY L, et al., 2020).

Existem também as fenocópias, ou seja, indivíduos que possuem alterações cardíacas semelhantes à $\mathrm{CMH}$, mas que não possuem mutações genéticas dos genes codificadores de proteínas dos sarcômeros. Na verdade, em tais casos estaremos diante de outras patologias, como cardiomiopatia LAMP2, PRKAG2, Doença de Fabry e amiloidose cardíaca (BAZAN SGZ, et al., 2020).

Importante destacar as diferenças ecocardiográficas existentes entre $\mathrm{CMH}$ e a Síndrome do coração do atleta. O diagnóstico diferencial é desafiador, porém fundamental, uma vez que vários estudos demonstraram que a principal causa de morte súbita em esportistas é a $\mathrm{CMH}$, seguida das anomalias de artérias coronárias (ALMEIDA SLAC, et al., 2021).

Já é de conhecimento que atletas de alto rendimento, em virtude da excessiva carga de treinamento a que são submetidos durante os períodos de preparo para competições, desenvolvem alterações cardíacas adaptativas, que podem variar de acordo com a modalidade que praticam. Algumas características ecocardiográficas encontradas no coração de atleta podem auxiliar no diagnóstico diferencial com $\mathrm{CMH}$ : diâmetro diastólico do VE de tamanho aumentado $>55 \mathrm{~mm}$; hipertrofia de distribuição simétrica; função diastólica normal; átrio esquerdo de tamanho normal; ausência de SAM e regressão da hipertrofia após destreinamento (GHORAYEB N, et al., 2019).

\section{Prognóstico}

Pacientes com $\mathrm{CMH}$ apresentam risco aumentado para morte súbita cardíaca por arritmias graves. Eles podem ser classificados em baixo, intermediário e alto risco, de acordo com a presença de fatores que implicam em pior prognóstico. A estratificação é fundamental para considerar a indicação de implante de cardiodesfibrilador implantável (CDI), que é o único método reconhecidamente eficaz em reduzir o risco de MSC. Geralmente esses pacientes apresentam uma boa evolução, com um risco estimado de cerca de 1\% ao ano, em comparação a 0,2\% com a população geral (MANDES L, et al., 2020).

Existem calculadoras capazes de estratificar o risco, dentre elas a utilizada pala European Society of Cardiology (ESC). A ecocardiografia também ganha importância nesse cenário, uma vez que das 7 condições associadas a maior risco de MSC, 3 são avaliadas pela ecocardiografia: gradiente da VSVE; tamanho do átrio esquerdo no plano paraestenal longitudinal; maior espessura miocárdica (TURVEY L, et al., 2020).

Espessuras superiores a $30 \mathrm{~mm}$ em qualquer segmento ventricular, correlacionam-se a riscos aumentados (MANDES L, et al., 2020). Já a relação entre o grau de obstrução da VSVE e MSC, possui evidências mais fracas em comparação aos outros achados. Outro fator que mais recentemente vem sendo implicado como preditor de MSC é a extensão da área de fibrose miocárdica, detectada através da ressonância magnética do coração pela técnica de realce tardio. Fibrose envolvendo mais de $15 \%$ da massa ventricular eleva o risco de arritmias ventriculares complexas (MARON MS, et al., 2017).

O risco aumentado para insuficiência cardíaca pode ser consequência da disfunção sistólica, disfunção diastólica e obstrução da VSVE. A fração de ejeção, parâmetro classicamente utilizado para avaliação da função sistólica, pode ser normal mesmo em indivíduos que já apresentam algum grau de disfunção pela técnica do Strain Longitudinal. Mesmo com essa limitação, a quantificação da fração de ejeção é importante, pois permite avaliar o risco de progressão para disfunção sistólica, que é maior quando atinge valores inferiores a $60 \%$ (TURVEY L, et al., 2020). 


\section{CONSIDERAÇÕES FINAIS}

A Cardiomiopatia Hipertrófica é uma condição genética que pode manifestar-se de forma assintomática ou evoluir com dor torácica, síncope, sinais e sintomas de insuficiência cardíaca e morte súbita cardíaca. Devido sua evolução potencialmente grave, o diagnóstico precoce é fundamental e tem como base a ecocardiografia transtorácica. Através desse exame, cada vez mais disponível nos serviços de saúde, podemos identificar e quantificar o espessamento parietal ventricular patológico, sua localização e outras condições associadas como obstrução da VSVE, insuficiência mitral, disfunção diastólica e comprometimento da função sistólica. Além disso, a ecocardiografia transtorácica também é uma ferramenta imprescindível para a estratificação dos pacientes em relação ao risco de morte súbita cardíaca. Alguns casos específicos podem necessitar de exames de ressonância magnética do coração para um diagnóstico mais preciso.

\section{REFERÊNCIAS}

1. ALMEIDA SLAC, et al. Morte súbita em atletas: Causas cardíacas. Brazilian Journal of Health Review. 2021; 4(2): 4592-4600.

2. BAZAN SGZ, et al. Cardiomiopatia Hipertrófica - Revisão. Arquivos Brasileiros de Cardiologia. 2020; 115(5): 927-935.

3. DOMINGUEZ F, et al. Role of echocardiography in the diagnosis and management of hypertrophic cardiomyopathy. Heart. 2017; 0: 1-13.

4. ELLIOTT PM, et al. 2014 ESC Guidelines on diagnosis and management of hypertrophic cardiomyopathy. European Heart Journal. 2014; 35: 2733-2779

5. FEIGENBAUM WA, RYAN T. Miocardioparia Hipertrófica e outras. Ecocardiografia. 2012; 19: 1069-1116.

6. GHORAYEB $\mathrm{N}$, et al. Atualização da Diretriz em Cardiologia do Esporte e do Exercício da Sociedade Brasileira de Cardiologia e da Sociedade Brasileira de Medicina do Exercício e Esporte. Arquivos Brasileiros de Cardiologia. 2019; 112(3): 326-368.

7. LOSI MA, et al. Echocardiography in patients with hypertrophic cardiomyopathy: usefulness of old and new techniques in the diagnosis and pathophysiological assessment. Cardiovascular Ultrasound. 2010, 8:7

8. MANDES L, et al. The role of echocardiography for diagnosis and prognostic stratification in hypertrophic cardiomyopathy. Journal of Echocardiography. 2020; 18: 137-148.

9. MARIAN AJ, BRAUNWALD E. Hypertrophic Cardiomyopathy Genetics, Pathogenesis, Clinical Manifestations, Diagnosis, and Therapy. Circulation Research. 2017; 121(7): 749-770.

10. MARON BJ. Clinical Course and Management of Hypertrophic Cardiomyopathy. The new englandjournal of medicine. 2018; 379: 655-68.

11. MARON MS, et al. How to Image Hypertrophic Cardiomyopathy. Circulation: Cardiovascular Imaging. 2017; 10:7

12. NAGUEH SF, et al. American Society of Echocardiography Clinical Recommendations for Multimodality Cardiovascular Imaging of Patients with Hypertrophic Cardiomyopathy. Jounal of The American Society of Echocardiography. 2011; 24: 473-98.

13. NAGUEH SF, et al. Recommendations for the Evaluation of Left Ventricular Diastolic Function by Echocardiography: An Update from the American Society of Echocardiography and the European Association of Cardiovascular Imaging. Journal of the American Society of Echocardiography. 2016; 29(4): 277-314.

14. QUADER N, et al. Cardiomiopatias. The Washington Manual of Echocardiography, second edition. 2018; 8: 93-102.

15. ROBINSON A, KRAMER CM. Imaging in Hypertrophic Cardiomyopathy. Journal of The American College of Cardiology. 2018; 4: 1-7.

16. SEMSARIAN C, et al. New Perspectives on the Prevalence of Hypertrophic Cardiomyopathy. Journal of The American College of Cardiology. 2015; 65(12): 1249-1254.

17. SEN-CHOWDHRY S, et al. Update on hypertrophic cardiomyopathy and a guide to the guidelines. Nature Reviews Cardiology. 2016; 13(11): 651-675.

18. STOLL CM, et al. Hypertrophic Cardiomyopathy Diagnosed on Point-of-Care Echocardiogram in an Elderly Patient With Syncope. Cureus. 2021; 13(8): e17008.

19. SUAIDE CES. Ecocardiografia Clínica e Cardiomiopatia Hipertrófica. Ecocardiografia: Princípios e Aplicações Clínicas. 2012; 35: 697-740.

20. TURVEY L, et al. Transthoracic echocardiography of hypertrophic cardiomyopathy in adults: a practical guideline from the British Society of Echocardiography. Echo Research and Practice. 2021; 8: 61-86

21. WILLIAMS LK, et al. Echocardiography in hypertrophic cardiomyopathy diagnosis, prognosis, and role in management. European Journal of Echocardiography. 2009; 10 (8): 9-14. 